\title{
Stereoselective entry into the D-GalNAc series starting from the D-Gal one: a new access to $\mathrm{N}$-acetyl-D-galactosamine and derivatives thereof ${ }^{*}$
}

\author{
Lorenzo Guazzelli, Giorgio Catelani*, Felicia D’Andrea, Alessia Giannarelli
}

Dipartimento di Chimica Bioorganica e Biofarmacia, Università di Pisa,

Via Bonanno, 33-I-56126, Pisa, Italy

\begin{abstract}
A new stereoselective preparation of $N$-aceyl-D-galactosamine (1b) starting from the know $p$ methoxyphenyl 3,4-O-isopropylidene-6- $O$-(1-methoxy-1-methylethyl)- $\beta$-D-galactopyranoside (10) is described using a simple strategy based on: (a) epimerization at C-2 of $\mathbf{1 0}$ via oxidation-reduction to give the talo derivative 11, (b) amination with configurational inversion at $\mathrm{C}-2$ of $\mathbf{1 1}$ via a $\mathrm{S}_{\mathrm{N}} 2$ on its 2-imidazylate, (c) anomeric deprotection of the $p$ methoxyphenyl $\beta$-D-galactosamine glycoside 14, (d) complete deprotection. Applying the same protocol to 2,3:5,6:3', 4'-tri- $O$-isopropylidene-6'-O-(1-methoxy-1-methylethyl)-lactose dimethyl acetal (4), directly obtained through acetonation of lactose, the disaccharide $\beta$-D-GalNAcp- $(1 \rightarrow 4)$-D-Glc $p(\mathbf{1 a})$ was obtained with complete stereoselectivity in good (40\%) overall yield from lactose.
\end{abstract}

Keywords: N-Acetyl-D-galactosamine, Amination with inversion, Epimerization, Lactose, $\beta$-D-Galactopyranosides

\section{Introduction}

D-Galactosamine (D-GalNH$)$ is, after D-glucosamine, the second most abundant natural aminosugar, isolated first in 1914 by Levene and La Forge ${ }^{2}$ from chondroitin, a mucopolysaccharide in which $\mathrm{D}-\mathrm{GalNH}_{2}$ is present, as in the structurally related dermatan sulfate, ${ }^{3}$ as $\mathrm{N}$-acetamido derivative (2-deoxy-2-acetamido-D-galactopyranose, D-GalNAc, 1b). D-GalNAc is also a constituent of several complex glycoproteins, being, for examples, the monosaccharide "core" of mucines, ${ }^{4}$ a component of oligosaccharide determinants of human blood group antigens, ${ }^{5}$ a constituent of anti-freeze glycoproteins of anthartic fishes. ${ }^{5}$ Furthermore, D-GalNAc has been recently recognised as one of the strongest agonist of the NKR-P1 rat Natural Killer cells receptor. ${ }^{6}$ Owing to the biological relevance of D-GalNAc, several approaches to its synthesis in the free form and/or to the synthesis of its derivatives have been proposed, using different carbohydrate starting materials. The two most exploited synthetic channels to D-GalNAc and its derivatives are those based (a) on the C-4 epimerization of the largely and cheaply available D-GlcNAc, ${ }^{7}$ mimicking thus the biosynthetic pathway, and (b) on the amination at C-2 of D-galactose with formal retention of configuration..$^{8-11}$ The first D-Gal to D-GalNAc transformation was described in $1976^{8}$ by Paulsen and co-workers using as key reaction the sodium azide opening of the epoxide ring of the intermediate 1,6:2,3-dianhydro- $\beta$-D-talopyranoside. Few years later, Lemieux and Ratcliffe ${ }^{9}$ used

\footnotetext{
FPart 25 of the series "Chemical Valorisation of Milk-derived Carbohydrates". For part 24, see Ref. 1.

Corresponding author. Tel.: +39 0502219700; fax: +39 0502219660; E-mail address: giocate@,farm.unipi.it (G. Catelani)
} 
the azidonitration of tri- $O$-acetyl-D-galactal for the synthesis of some 2-azido-2-deoxy-Dgalactopyranose derivatives and free D-GalNAc (1b). After this key paper, several addition reactions to D-galactal derivatives have been proposed to obtain D-GalNAc and its glycosides, as, the Danishefsky's sulfamidoglycosylation, ${ }^{10}$ the Gin's acetamidoglycosylation. ${ }^{11}$ Furthermore, specific synthesis of $\mathrm{D}_{-}-\mathrm{GalNH}_{2}$ starting from $\mathrm{L}-\mathrm{lyxose}{ }^{12}$ and, very recently, $\mathrm{D}$-tagatose ${ }^{13}$ have been proposed.

In the frame of a general project on the synthesis of $\beta$-D-hexosaminyl-( $1 \rightarrow 4)$-D-Glc $p$ disaccharides, ${ }^{14}$ we considered the synthesis of $\beta$-D-GalNAc $p-(1 \rightarrow 4)-D-G l c p$ (1a), a natural disaccharide present in minute amount in the bovine colostrum. ${ }^{15}$ The sole reported synthesis of $\mathbf{1 a}$ is based on an enzymatic glycosylation involving a $\beta-1 \rightarrow 4-N$-acetylgalactosaminyltransferase. ${ }^{16}$ However, the above synthesis do not appears suitable for preparative purposes. Presented herein is a new efficient chemical method for the preparation of the disaccharide 1a starting from lactose, involving a two-steps amination with overall retention of configuration at C-2' (Chart 1), through the formation of $\beta$-D-talopyranoside intermediate of type 2 . The potentiality of the method has also been demonstrated in the case of a monosaccharide $\beta$-D-galactopyranoside, that has been transformed into a know precursor of free D-GalNAc (1) and of some 2-azido-2-deoxy-Dgalactopyranoside glycosyl donors.

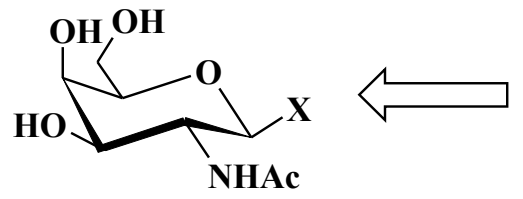

1a: $X=4-O$-D-glucopyranosyl

1b: $\mathbf{X}=\mathbf{O H}$

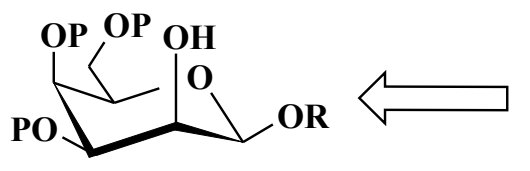

2

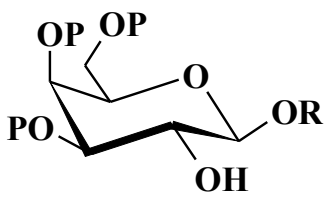

3

Chart 1: Retrosynthetic approach for transforming a $\beta$-D-galactopyranoside unit into a D-GalNAc one.

\section{Results and Discussion}

A straightforward route to $\beta$-D-GalNAcp-(1 $\rightarrow 4)-\mathrm{D}-\mathrm{Glc} p$ (1a) was envisaged (Scheme 1) taking advantage from the easily availability of the tetraacetonide $5,{ }^{17}$ prepared from its $\mathrm{C}-2$ ' lactose epimer (4) through a completely stereoselective, high yielding (92\%) oxidation-reduction sequence. ${ }^{17}$ The activation of 5 was made by treatment with imidazyl sulfate $\left(\operatorname{Im}_{2} \mathrm{SO}_{2}\right)$ and $\mathrm{NaH}$ in DMF at $-30{ }^{\circ} \mathrm{C}$, leading to the corresponding imidazylate 6 , isolated pure by flash chromatography in $86 \%$ yield. 6 was then subjected to a $\mathrm{S}_{\mathrm{N}} 2$ substitution with $\mathrm{NaN}_{3}$ in $\mathrm{DMF}$ at $100{ }^{\circ} \mathrm{C}$, obtaining, after flash chromatography, the azido derivative 7 in $94 \%$ yield. This result confirms the usefulness of the imidazylate leaving group for performing efficient substitution in position 2 of a pyranoside, 
where other aryl and alkyl sulfonates are known to give unsatisfactory results. ${ }^{18}$
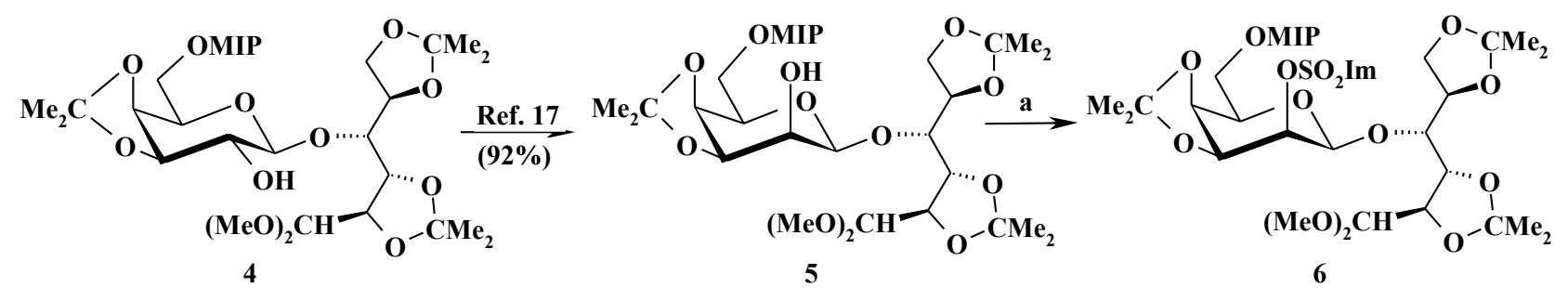

6
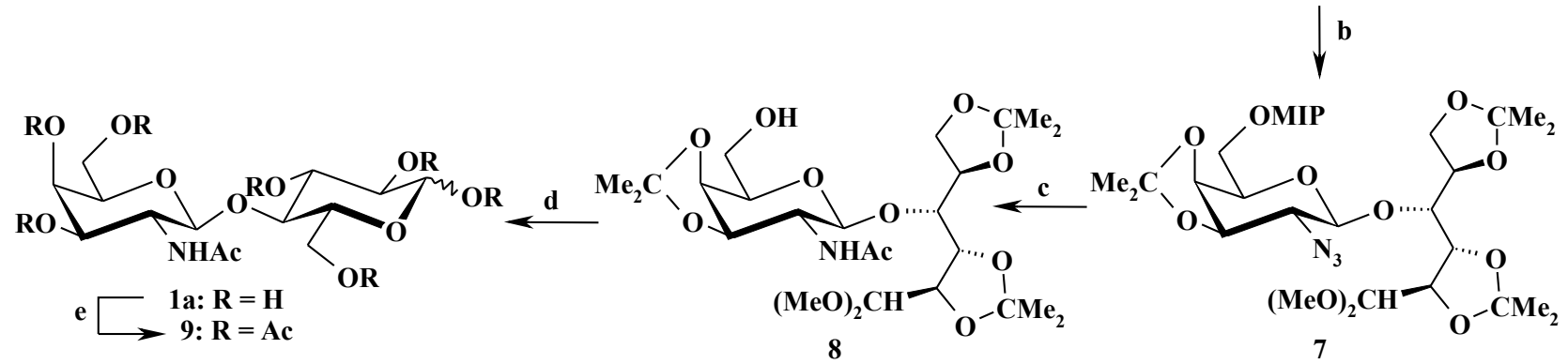

MIP $=-\mathrm{C}(\mathrm{OMe}) \mathrm{Me}_{2}$

Scheme 1: Synthesis of $\beta$-D-GalNAcp-(1-->4)-D-Glcp disaccharide from lactose. Reagents and conditions: (a) $\operatorname{Im}_{2} \mathrm{SO}_{2}, \mathrm{NaH}, \mathrm{DMF},-30{ }^{\circ} \mathrm{C}$, $4 \mathrm{~h}$ (86\%); (b) $\mathrm{NaN}_{3}, \mathrm{DMF}, 100{ }^{\circ} \mathrm{C}, 1.2 \mathrm{~h}$ (94\%); (c) (1) $\mathrm{NiCl}_{2} \cdot 6 \mathrm{H}_{2} \mathrm{O}, \mathrm{NaBH}_{4}, \mathrm{MeOH}, 0{ }^{\circ} \mathrm{C}$, room temp, $2 \mathrm{~h}$; (2) $\mathrm{Ac} \mathrm{O}_{2} \mathrm{O}, \mathrm{MeOH}$, room temp, $2 \mathrm{~h}(86 \%)$; (d) $80 \%$ aq $\mathrm{AcOH}, 80^{\circ} \mathrm{C}, 4 \mathrm{~h}(90 \%)$; (e) $\mathrm{Ac}_{2} \mathrm{O}$, Py, room temp, $18 \mathrm{~h}(95 \%)$.

The reduction of the azido group of 7 employing nickel chloride hexahydrate and sodium borohydride, afforded a single product which was directly submitted to $N$-acetylation $\left(\mathrm{Ac}_{2} \mathrm{O}\right.$ in $\mathrm{MeOH})$. In the slightly acid reaction conditions the labile 6'-mixed acetal group was removed and compound 8 was obtained in $86 \%$ yield, after chromatographic purification. The target compound 1a was instead prepared by complete deprotection of 8 using $80 \%$ aq $\mathrm{AcOH}$ at $80{ }^{\circ} \mathrm{C}$ : the reaction involves the O-deisopropylidenation and the C-1 aldehydo group exposition with contemporary concomitant six membered ring closure. The structure of $\mathbf{1 a}$ as well as its anomeric composition $(\alpha /$ $\beta$ ratio about 2:3) were established on the basis of its NMR spectra. In particular, the ${ }^{1} \mathrm{H}$ NMR spectrum was identical to the reported one, ${ }^{15}$ while the ${ }^{13} \mathrm{C}$ NMR signals, not yet reported, were assigned by comparison (see Table 1 ) with those of $\alpha$ - and $\beta$-lactose, ${ }^{19}$ for the gluco portion, and those of methyl 2-acetamido-2-deoxy- $\beta$-D-galactopyranoside ${ }^{20}$ (Me $\beta$-D-GalNAc, not showed). $\mathrm{NMR}$ analysis of the acetylated disaccharide 9, obtained treating $\mathbf{1 a}$ with $\mathrm{Ac}_{2} \mathrm{O}$ in pyridine for $18 \mathrm{~h}$, further confirmed the parent compound structure. It is worth of note that the transformation of lactose into the 2'-aminated analogue is obtained with a 7 steps process involving common reagents and simple manipulations in a very good overall yield (40\%), certainly not easily achieved through chemical means starting from the two deprotected monosaccharide components. On the basis of this positive result, the same protocol of $\mathrm{C}-2$ amination with retention of configuration was applied (Scheme 2) to $p$-methoxyphenyl 3,4-O-isopropylidene-6-O-(1-methoxy-1-methylethyl)- $\beta$-Dgalactopyranoside (10), easily obtainable from commercial penta- $O$-acetyl- $\beta$-D-galactopyranose 
through a simple sequence. ${ }^{21}$ Also in this case, the introduction of the azido group in position 2 was achieved by an high yielding double stereoselective inversion sequence, providing first the preparation of the talo-derivative 11 through an oxidation at C-2 (TPAP-NMO) followed by reduction of the crude uloside with $\mathrm{NaBH}_{4}$ in $\mathrm{MeOH}$. The stereoselectivity of the reduction was again complete leading to the exclusive formation of $\mathbf{1 1}$ ( $81 \%$ isolated yield) due to the $\beta$ face shielding of the acetonide bridge, which completely inhibits the hydride attack on the same face. The axial hydroxyl function was then activated as sulfonyl imidazole $\left(\mathrm{Im}_{2} \mathrm{SO}_{2}\right.$, and $\mathrm{NaH}$ in DMF at $-30{ }^{\circ} \mathrm{C}$ ) and 12 was obtained in 92\% yield after chromatographic purification. The second inversion-amination was performed, as described for the disaccharide analogue, by treatment with $\mathrm{NaN}_{3}$ in DMF at $100{ }^{\circ} \mathrm{C}$, obtaining the desired azido derivative $\mathbf{1 3}$ in $96 \%$ yield. The two consecutive inversion of configuration were easily checked by ${ }^{1} \mathrm{H} \mathrm{NMR}$, on the basis of $J_{1,2}$ and $J_{2,3}$ values, 2.1 and $4.5 \mathrm{~Hz}$ respectively for the talo derivative 11, and 8.6 and 7.5 for the galacto one 13.

Although some reported methods ${ }^{22}$ enable the direct transformation of the anomeric $p$-methoxyphenyl into other more reactive leaving groups, our next target was the known azido derivative $\mathbf{1 5}^{23}$ and its peracetylated derivative $\mathbf{1 6} .^{24}$

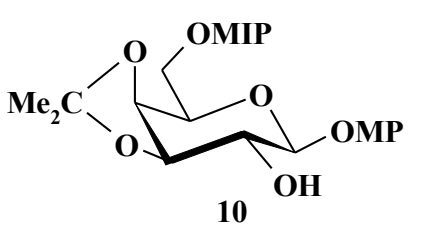

$\mathbf{M P}=p$-methoxyphenyl

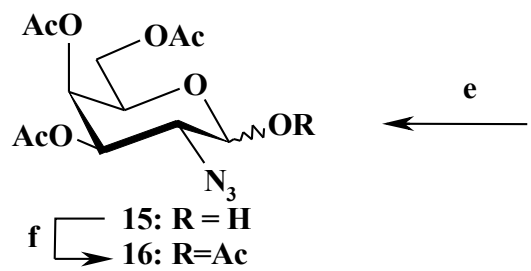

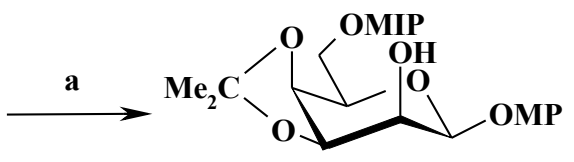

11

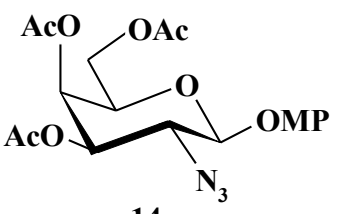

14

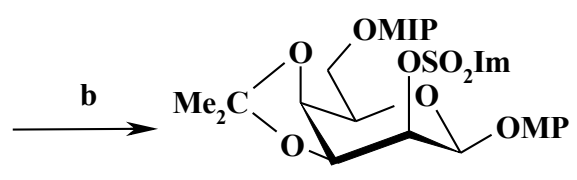

12

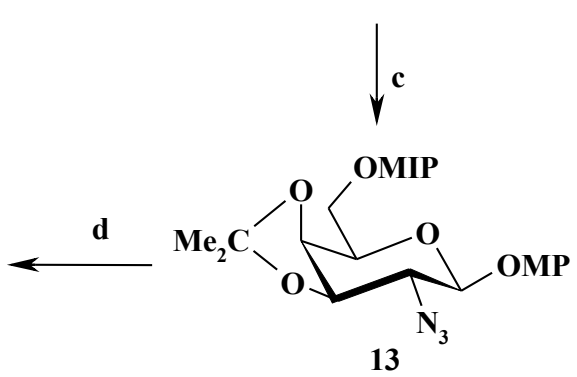

Scheme 2: Formal synthesis of D-GalNAc and of 2-deoxy-2-azido-D-galactopyranosyl glycosyl donors. Reagents and conditions: (a) (1) TPAP, NMO, $\mathrm{CH}_{2} \mathrm{Cl}_{2}$, room temp, $2 \mathrm{~h}$; (2) $\mathrm{NaBH}_{4}, \mathrm{MeOH}, 0^{\circ} \mathrm{C}, 2 \mathrm{~h}$ (81\% overall); (b) $\mathrm{Im}_{2} \mathrm{SO}_{2}, \mathrm{NaH}, \mathrm{DMF},-30{ }^{\circ} \mathrm{C}, 20 \mathrm{~min}$ (92\%); (c) $\mathrm{NaN}_{3}, \mathrm{DMF}, 100{ }^{\circ} \mathrm{C}, 2 \mathrm{~h}$ (96\%); (d) (1) $80 \%$ aq AcOH, $80{ }^{\circ} \mathrm{C}, 2 \mathrm{~h}$; (2) Ac $2 \mathrm{O}$, Py, room temp, $2 \mathrm{~h}$ (97\% overall); (e) CAN, 3:1 $\mathrm{Me}_{2} \mathrm{CO}-\mathrm{H}_{2} \mathrm{O}, 0{ }^{\circ} \mathrm{C}$, room temp, $30 \mathrm{~min}(81 \%)$; (f) $\mathrm{Ac}_{2} \mathrm{O}$, Py, room temp, $15 \mathrm{~h}(95 \%)$.

This goal was achieved by a first removal of the acetal functions by acid hydrolysis with $80 \%$ aq $\mathrm{AcOH}$ and acetylation of the crude residue $\left(\mathrm{Ac}_{2} \mathrm{O}-\mathrm{Py}\right)$, obtaining compound $\mathbf{1 4}$ in almost quantitative yield (97\%) after chromatographic purification. The removal of the anomeric $p$ methoxy phenyl was performed with CAN, leading to isolate ( $81 \%$ yield) the reducing derivative $\mathbf{1 5}$, that was, finally, quantitatively transformed into the peracetate $\mathbf{1 6}$ by routine acetylation. 
The transformation of $\alpha-16$ into D-GalNAc hydrochloride has been described by Lemieux, ${ }^{9}$ while 15 constitutes the key precursor of a series of 2-azido-2-deoxy-D-galactopyranose glycosyl donors. ${ }^{25}$

In conclusion, we have proposed an easy D-galactose into D-galactosamine transformation with complete stereoselectivity avoiding thus difficult diastereoisomeric separations. Using this new methodology the first chemical synthesis of the $\beta$-D-GalNAc $p-(1 \rightarrow 4)$-D-Glc $p$ disaccharide has been achieved with excellent yield starting from lactose, while in the monosaccharide series, a new synthesis of D-GalNAc and of some 2-azido-2-deoxy-D-galactopyranosyl donors has been accomplished, complementing thus the existing approaches. This strategy of amination with overall retention of configuration (double inversion) gave good results both in the mono- and disaccharide series and seems to have general applicability and good potentiality in the construction of complex $\beta$-D-GalNAc containing oligosaccharides through a first $\beta$-galactosylation followed by its $\mathrm{C}-2$ amination with retention of configuration.

\section{Experimental}

\subsection{General methods.}

General methods are those reported in Ref. $1 .{ }^{1} \mathrm{H}$ NMR and ${ }^{13} \mathrm{C}$ NMR spectra were recorded with an Avance II 250 spectrometer operating at $250.13 \mathrm{MHz}\left({ }^{1} \mathrm{H}\right)$ and $62.9 \mathrm{MHz}\left({ }^{13} \mathrm{C}\right)$ in the reported solvent (internal standard $\mathrm{Me}_{4} \mathrm{Si}$ ) and the assignments were made, when possible, with DEPT, HETCOR and COSY experiments. Compounds $\mathbf{5}^{17}$ and $\mathbf{1 0}^{21}$ were prepared according to described procedures.

\subsection{4-O-[2-O-(1-imidazolylsulfonyl)-3,4-O-isopropylidene-6-O-(1-methoxy-1-methylethyl)- $\beta$-D- talopyranosyl]-2,3:5,6-di-O-isopropylidene-aldeydo-D-glucose dimethyl acetal (6).}

To a suspension of $60 \% \mathrm{NaH}$ in mineral oil $(632 \mathrm{mg}, 15.8 \mathrm{mmol})$ pre-washed with hexane under argon atmosphere and cooled to $0{ }^{\circ} \mathrm{C}$, a soln of $\mathbf{5}^{17}(1.84 \mathrm{~g}, 3.17 \mathrm{mmol})$ in dry DMF (55 mL) was slowly added. The mixture was stirred at $0{ }^{\circ} \mathrm{C}$ for $30 \mathrm{~min}$, cooled to $-30{ }^{\circ} \mathrm{C}$, treated with $\operatorname{Im}_{2} \mathrm{SO}_{2}$ (940 mg, $4.74 \mathrm{mmol}$ ) and further stirred until TLC analysis (EtOAc) revealed the complete disappearance of the starting material $(4 \mathrm{~h})$. The reaction mixture was then cooled to $-40{ }^{\circ} \mathrm{C}$, excess of $\mathrm{NaH}$ was destroyed by addition of $\mathrm{MeOH}(0.5 \mathrm{~mL})$ followed by $10 \mathrm{~min}$ stirring, and partitioned between $\mathrm{Et}_{2} \mathrm{O}(50 \mathrm{~mL})$ and crushed iced-water. The organic phase was separated, and the aq layer extracted with $\mathrm{Et}_{2} \mathrm{O}(4 \times 50 \mathrm{~mL})$. The collected organic phases were dried $\left(\mathrm{MgSO}_{4}\right)$, filtered and concentrated at diminished pressure. The flash chromatographic purification over silica gel of the 
reaction product, eluting with 1:3 hexane-EtOAc $+0.1 \%$ of $\mathrm{Et}_{3} \mathrm{~N}$, gave pure $6(1.93 \mathrm{~g}, 86 \%)$ as a syrup; $[\alpha]_{\mathrm{D}}+2.7$ (c 1.06, $\left.\mathrm{CHCl}_{3}\right) ; R_{\mathrm{f}} 0.33\left(1: 3\right.$ hexane-EtOAc); ${ }^{1} \mathrm{H} \mathrm{NMR}\left(\mathrm{CD}_{3} \mathrm{CN}\right): \delta 7.98(\mathrm{dd}, 1 \mathrm{H}$, $\left.J_{2,4} 1.3 \mathrm{~Hz}, J_{2,5} 0.9 \mathrm{~Hz}, \mathrm{Im}-\mathrm{H}-2\right), 7.44$ (dd, 1H, $\left.J_{4,5} 1.7 \mathrm{~Hz}, \mathrm{Im}-\mathrm{H}-4\right), 7.05$ (dd, 1H, Im-H-5), 4.92 (dd, $1 \mathrm{H}, J_{1^{\prime}, 2}, 1.0 \mathrm{~Hz}, J_{2}, 3^{\prime}, 5.7 \mathrm{~Hz}, \mathrm{H}-2$ '), 4.82 (d, 1H, H-1'), 4.40 (dd, 1H, $J_{3^{\prime},{ }^{\prime}}, 5.8 \mathrm{~Hz}, \mathrm{H}-3$ '), 4.36 (d, 1H, $\left.J_{1,2} 6.0 \mathrm{~Hz}, \mathrm{H}-1\right), 4.27$ (dd, 1H, $\left.J_{2,3} 7.7 \mathrm{~Hz}, \mathrm{H}-2\right), 4.15$ (dt, $1 \mathrm{H}, J_{4,5} 5.9 \mathrm{~Hz}, J_{5,6 \mathrm{a}}=J_{5,6 \mathrm{~b}} 6.1 \mathrm{~Hz}, \mathrm{H}-5$ ), 4.12 (dd, 1H, J',5, 2.7 Hz, H-4'), 4.04 (dd, 1H, J3,4 1.9 Hz, H-3), 3.94 (dd, 1H, J6a,6b 8.6 Hz, H-6b), 3.84 (dd, 1H, H-6a), 3.80 (dd, 1H, H-4), 3.80 (ddd, 1H, $J_{5^{\prime}, 6^{\prime} \mathrm{a}} 6.5$ Hz, $J_{5^{\prime}, 6^{\prime} \mathrm{b}} 6.3 \mathrm{~Hz}, \mathrm{H}-5$ '), 3.63 (dd,

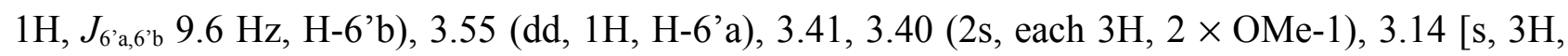
$\left.\mathrm{C}(\mathrm{OMe}) \mathrm{Me}_{2}\right], 1.37,1.36,1.32,1.30,1.29,1.28\left(6 \mathrm{~s}\right.$, each $\left.3 \mathrm{H}, 3 \times \mathrm{CMe}_{2}\right), 1.22,1.20$ [2s, each $3 \mathrm{H}$, $\left.\mathrm{C}(\mathrm{OMe}) \mathrm{Me}_{2}\right] ;{ }^{13} \mathrm{C} \mathrm{NMR}\left(\mathrm{CD}_{3} \mathrm{CN}\right): \delta 138.1$ (Im-C-2), 130.9 (Im-C-5), 120.1 (Im-C-4), 110.7, 110.3, $109.2\left(3 \times C \mathrm{Me}_{2}\right), 106.5(\mathrm{C}-1), 100.9$ [C(OMe)Me 2 ], $99.1(\mathrm{C}-1$ '), 79.9 (C-2'), 78.5 (C-4), 77.9 (C-3), 77.1 (C-5), 76.3 (C-2), 73.5 (C-5'), 71.9 (C-3’), 71.1 (C-4'), 66.5 (C-6), 60.3 (C-6’), 56.6, $54.7(2 \times \mathrm{OMe}-1), 48.9\left[\mathrm{C}(\mathrm{OMe}) \mathrm{Me}_{2}\right], 27.3,27.1,27.0,25.8,25.4,25.3\left(3 \times \mathrm{CMe}_{2}\right), 24.7,24.6$ [C(OMe)Me $\mathrm{Me}_{2}$. Anal Calcd for $\mathrm{C}_{30} \mathrm{H}_{50} \mathrm{~N}_{2} \mathrm{O}_{15} \mathrm{~S}: \mathrm{C}, 50.69 ; \mathrm{H}, 7.09 ; \mathrm{N}, 3.94$. Found: C, 50.89; H, 7.43; N, 5.29.

\subsection{4-O-[2-azido-2-deoxy-3,4-O-isopropylidene-6-O-(1-methoxy-1-methylethyl)- $\beta$-D- galactopyranosyl]-2,3:5,6-di-O-isopropylidene-aldeydo-D-glucose dimethyl acetal (7).}

A soln of 6 (3.85 g, $5.41 \mathrm{mmol})$ and $\mathrm{NaN}_{3}(707 \mathrm{mg}, 10.9 \mathrm{mmol})$ in dry DMF (100 mL) was stirred under argon atmosphere at $100^{\circ} \mathrm{C}$. After $1 \mathrm{~h}$ and $20 \mathrm{~min}$ TLC analysis (EtOAc) revealed the complete disappearance of the starting material, the mixture was cooled to room temp and partitioned between satd aq $\mathrm{NaHCO}_{3}(50 \mathrm{~mL})$ and $\mathrm{Et}_{2} \mathrm{O}(50 \mathrm{~mL})$. The organic phase was separated and the aq layer extracted with $\mathrm{Et}_{2} \mathrm{O}(3 \times 50 \mathrm{~mL})$. The organic extracts were dried $\left(\mathrm{MgSO}_{4}\right)$, filtered and concentrated at diminished pressure. Purification of the residue by flash chromatography over silica gel $\left(7: 3\right.$ hexane-EtOAc $+0.1 \%$ of $\left.\mathrm{Et}_{3} \mathrm{~N}\right)$ gave pure $7(3.08 \mathrm{~g}, 94 \%)$ as a clear syrup; $[\alpha]_{\mathrm{D}}-30.0$ (c 1.0, $\left.\mathrm{CHCl}_{3}\right) ; R_{\mathrm{f}} 0.64$ (EtOAc); ${ }^{1} \mathrm{H}$ NMR $\left(\mathrm{CD}_{3} \mathrm{CN}\right): \delta 4.63\left(\mathrm{~d}, 1 \mathrm{H}, J_{1^{\prime}, 2^{\prime}}, 8.5 \mathrm{~Hz}, \mathrm{H}-1\right.$ '), $4.34(\mathrm{~d}, 1 \mathrm{H}$, $\left.J_{1,2} 6.0 \mathrm{~Hz}, \mathrm{H}-1\right), 4.32$ (t, 1H, $\left.J_{2,3} 6.0 \mathrm{~Hz}, \mathrm{H}-2\right), 4.25$ (dt, $\left.1 \mathrm{H}, J_{4,5} 4.7 \mathrm{~Hz}, J_{5,6 \mathrm{a}}=J_{5,6 \mathrm{~b}} 6.4 \mathrm{~Hz}, \mathrm{H}-5\right), 4.15$ (dd, $1 \mathrm{H}, J_{3^{\prime}, 4^{\prime}}, 5.4 \mathrm{~Hz}, J_{4^{\prime}, 5}, 2.1 \mathrm{~Hz}, \mathrm{H}-4$ '), 4.09 (dd, 1H, $J_{6 \mathrm{a}, 6 \mathrm{~b}} 8.5 \mathrm{~Hz}, \mathrm{H}-6 \mathrm{~b}$ ), 4.06 (dd, 1H, J3,4 $1.3 \mathrm{~Hz}$,

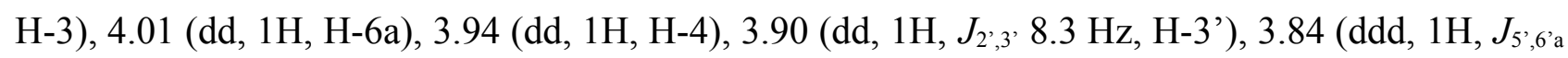

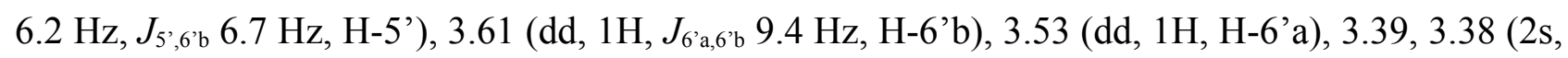
each 3H, $2 \times \mathrm{OMe}), 3.30\left(\mathrm{dd}, 1 \mathrm{H}, \mathrm{H}-2\right.$ ') 3.15 [s, 3H, C(OMe) $\left.\mathrm{Me}_{2}\right], 1.49,1.40,1.33,1.32,1.30$, 1.29 (6s, each 3H, $\left.3 \times \mathrm{CMe}_{2}\right), 1.29,1.30$ [2s, each $\left.3 \mathrm{H}, \mathrm{C}(\mathrm{OMe}) \mathrm{Me}_{2}\right] ;{ }^{13} \mathrm{C} \mathrm{NMR}\left(\mathrm{CD}_{3} \mathrm{CN}\right): \delta 110.9$, 110.7, $108.9\left(3 \times C \mathrm{Me}_{2}\right), 106.4(\mathrm{C}-1), 102.2\left(\mathrm{C}-1\right.$ '), 100.9 [C(OMe)Me $\mathrm{Me}_{2}$, 78.5 (C-3), $77.9(\mathrm{C}-5)$, 
77.8 (C-3'), 76.7 (C-2), 76.2 (C-4), 73.8 (C-4'), 73.0 (C-5'), 67.3 (C-2'), 66.0 (C-6), 60.4 (C-6'), 56.3, $54.4(2 \times \mathrm{OMe}), 48.9\left[\mathrm{C}(\mathrm{OMe}) \mathrm{Me}_{2}\right] ; 28.4,27.6,27.1,26.7,26.3,25.2\left(3 \times \mathrm{CMe}_{2}\right), 24.7,24.5$ $\mathrm{C}(\mathrm{OMe}) \mathrm{Me}_{2}$ ]. Anal Calcd for $\mathrm{C}_{27} \mathrm{H}_{47} \mathrm{~N}_{3} \mathrm{O}_{12}: \mathrm{C}, 53.54 ; \mathrm{H}, 7.82 ; \mathrm{N}, 6.94$. Found: C, 53.47; H, 7.62; N, 7.12 .

\subsection{4-O-(2-acetamido-2-deoxy-3,4- $O$-isopropylidene- $\beta$-D-galactopyranosyl)-2,3:5,6-di- $O$ - isopropylidene-aldeydo-D-glucose dimethyl acetal (8).}

To a soln of $7(1.40 \mathrm{~g}, 2.31 \mathrm{mmol})$ in $\mathrm{MeOH}(24 \mathrm{~mL})$ cooled to $0{ }^{\circ} \mathrm{C}, \mathrm{NiCl}_{2} \cdot 6 \mathrm{H}_{2} \mathrm{O}(2.75 \mathrm{~g}, 11.5$ mmol) and $\mathrm{NaBH}_{4}(699 \mathrm{mg}, 18.4 \mathrm{mmol})$ were added. The soln was warmed to room temp and stirred for $2 \mathrm{~h}$. To the mixture were then added brine $(50 \mathrm{~mL})$ and, after $10 \mathrm{~min}$, water $(50 \mathrm{~mL})$ and $\mathrm{CHCl}_{3}(50 \mathrm{~mL})$. The organic phase was separated and the aq layer extracted with $\mathrm{CHCl}_{3}(4 \times 50$ $\mathrm{mL})$. The collected organic extracts were dried $\left(\mathrm{MgSO}_{4}\right)$, filtered and concentrated at diminished pressure. The residue was dissolved in $\mathrm{MeOH}(30 \mathrm{~mL}), \mathrm{Ac}_{2} \mathrm{O}(7.5 \mathrm{~mL})$ was added and the mixture was stirred at room temp for $2 \mathrm{~h}$ when TLC analysis (EtOAc) showed the formation of a new product. The reaction mixture was repeatedly co-evaporated with toluene $(4 \times 30 \mathrm{~mL})$ under diminished pressure and purified by flash chromatography over silica gel $\left(49: 1 \mathrm{CHCl}_{3}-\mathrm{MeOH}\right)$ affording pure $8(1.09 \mathrm{~g}, 86 \%)$ as a clear syrup; $[\alpha]_{\mathrm{D}}+8.15(\mathrm{c} 1.46, \mathrm{MeOH}) ; R_{\mathrm{f}} 0.10\left(\right.$ EtOAc); ${ }^{1} \mathrm{H}$

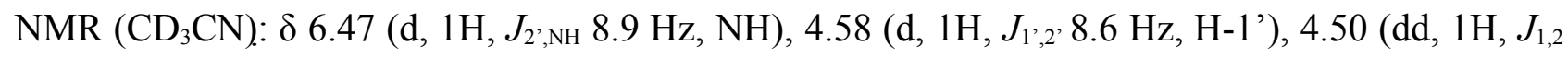
6.9 Hz, J2,3 7.0 Hz, H-1), 4.34 (d, 1H, H-1), 4.36-4.05 (m, 4H, H-5, H-3', H-6'a, H-6'b), 3.97-3.70 (m, 4H, H-5', H-3, H-6a, H-6b), 3.51 (m, 2H, H-2, H-4), 3.43, 3.42 (2s, each 3H, $2 \times \mathrm{OMe}), 1.90$ (s,

$3 \mathrm{H}, \mathrm{MeCON}), 1.45,1.40,1.32,1.31,1.28,1.26\left(6 \mathrm{~s}\right.$, each $\left.3 \mathrm{H}, 3 \times \mathrm{CMe}_{2}\right) ;{ }^{13} \mathrm{C} \mathrm{NMR}\left(\mathrm{CD}_{3} \mathrm{CN}\right): \delta$ $170.6(\mathrm{MeCO}), 110.2,110.0,108.5\left(3 \times \mathrm{CMe}_{2}\right), 107.6(\mathrm{C}-1), 101.6(\mathrm{C}-1$ '), $78.6(\mathrm{C}-3), 77.4,77.3$ (C-3', C-5), 75.7, 75.6 (C-4, C-2), 74.6 (C-4’), 73.5 (C-5'), 65.6 (C-6), 62.5 (C-6'), 57.5, 54.6 (2 × OMe), 55.2 (C-2'), 28.2, 27.4, 26.8, 26.4, 26.3, 24.8 (3× $\left.\mathrm{CMe}_{2}\right), 23.3$ ( $\left.\mathrm{MeCON}\right)$. Anal Calcd for $\mathrm{C}_{25} \mathrm{H}_{43} \mathrm{NO}_{12}$ : C, 54.63; H, 7.89; N, 2.55. Found: C, 54.72; H, 7.91; N, 2.58.

\subsection{4- $O$-(2-Acetamido-2-deoxy- $\beta$-D-galactopyranosyl]- $\alpha, \beta$-D-glucopyranose (1a).}

A soln of $8(450 \mathrm{mg}, 0.82 \mathrm{mmol})$ in $80 \%$ aq $\mathrm{AcOH}(15 \mathrm{~mL})$ was stirred at $80{ }^{\circ} \mathrm{C}$ for $4 \mathrm{~h}$ and then concentrated at diminished pressure by co-evaporation with toluene $(4 \times 35 \mathrm{~mL})$. The residue was triturated with EtOAc to give an amorphous white solid (283 mg, 90\%) constituted $\left({ }^{13} \mathrm{C} \mathrm{NMR,} \mathrm{D}_{2} \mathrm{O}\right)$ by a 2:3 $\alpha / \beta$ anomeric mixture of 1a, as established on the basis of the integration of the H-1 signals; $[\alpha]_{\mathrm{D} \infty}=+55.8$ (c 0.92, water); selected ${ }^{1} \mathrm{H} \operatorname{NMR}\left(\mathrm{D}_{2} \mathrm{O}\right)$ data of $\boldsymbol{\alpha}-\mathbf{1 a}: \delta 5.21\left(\mathrm{~d}, 1 \mathrm{H}, J_{1,2} 3.8 \mathrm{~Hz}, \mathrm{H}-1\right)$, 
$4.52\left(\mathrm{~d}, 1 \mathrm{H}, J_{1^{\prime}, 2}, 8.4 \mathrm{~Hz}, \mathrm{H}-1^{\prime}\right)$; $\boldsymbol{\beta}-1 \mathbf{a}: \delta 4.65$ (d, $\left.1 \mathrm{H}, J_{1,2} 8.4 \mathrm{~Hz}, \mathrm{H}-1\right), 4.51$ (d, 1H, $\left.J_{1^{\prime}, 2}, 8.3 \mathrm{~Hz}, \mathrm{H}-1^{\prime}\right)$, $3.27\left(\mathrm{dd}, 1 \mathrm{H}, J_{2,3} 8.8 \mathrm{~Hz}, \mathrm{H}-2\right) ;{ }^{13} \mathrm{C}$ NMR $\left(\mathrm{D}_{2} \mathrm{O}\right)$ data of $\boldsymbol{\alpha}-\mathbf{1 a}$ and $\boldsymbol{\beta}-\mathbf{1 a}$ see Table 1 and $\delta$ : 177.6 (MeCO), 25.0 (MeCO). Anal. Calcd for $\mathrm{C}_{14} \mathrm{H}_{25} \mathrm{NO}_{11}$ : C, 43.86; H, 6.57; N, 3.65. Found: C, 43.95; H, 6.59; N, 3.66.

\subsection{4- $O$-(2-Acetamido-2-deoxy-3,4,6-tri- $O$-acetyl- $\beta$-D-galactopyranosyl]- $\alpha, \beta-1,2,3,6$-tetra- $O$ - acetyl-D-glucopyranose (9).}

1 a $(50 \mathrm{mg}, 0.13 \mathrm{mmol})$ was dissolved in a $2: 1$ pyridine- $\mathrm{Ac}_{2} \mathrm{O}$ solution $(3 \mathrm{~mL})$ and the resulting soln was stirred at room temp for $18 \mathrm{~h}$ and then co-evaporated with toluene $(3 \times 5 \mathrm{~mL})$ under diminished pressure. The flash chromatographic purification, eluting with EtOAc, afforded pure 9 (84 mg, 95\%) as an 1:1 $\alpha / \beta$ anomeric mixture, as established on the basis of the integration of the $\mathrm{H}-1$ signals. 9 was a syrup; $[\alpha]_{\mathrm{D}}+16.0\left(\mathrm{c} 1.04, \mathrm{CHCl}_{3}\right) ; R_{\mathrm{f}} 0.23(\mathrm{EtOAc}) ;{ }^{1} \mathrm{H} \mathrm{NMR}\left(\mathrm{CD}_{3} \mathrm{CN}\right)$ of $\boldsymbol{\alpha}-9$ :

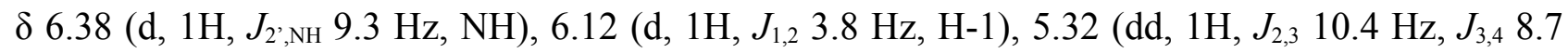

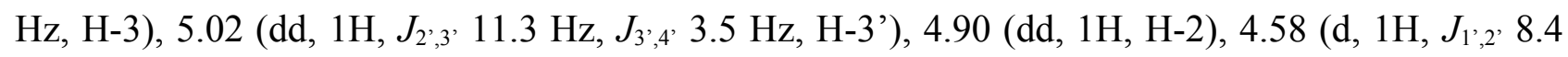
Hz, H-1'), 1.82 (s, 3H, MeCON); $\beta-9: \delta 6.36$ (d, 1H, $J_{2}$,NH $\left.9.3 \mathrm{~Hz}, \mathrm{NH}\right), 5.74$ (d, 1H, $J_{1,2} 8.3 \mathrm{~Hz}$, H-1), 5.24 (m, 1H, H-3), 5.03 (dd, 1H, $J_{2}, 3,11.2$ Hz, J', ${ }^{\prime}, 3.5$ Hz, H-3'), 4.92 (dd, 1H, J2,3 9.7 Hz, H-2), 4.59 (d, 1H, $J_{1}, 2,8.4 \mathrm{~Hz}, \mathrm{H}-1$ '), 1.83 (s, 3H, MeCON); cluster of signals for both anomers: $\delta$ 4.40-3.95 (m, 14H, H-4, H-5, H-6a, H-6b, H-5', H-6'a, H-6’b), 3.86 (m 2H, H-2') 2.13, 2.08, 2.07, 2.06, 2.05, 2.04, 2.03, 2.02, 2.01, 2.00, 1.98, 1.97, 1.96, $1.90(14 \mathrm{~s}$, each $3 \mathrm{H}, 14 \mathrm{x} \mathrm{MeCOO}) ;{ }^{13} \mathrm{C}$ NMR ( $\left.\mathrm{CD}_{3} \mathrm{CN}\right) \boldsymbol{\alpha}-9:$ : 102.1 (C-1'), 89.6 (C-1), 76.1 (C-4), 71.3 (C-5, C-5'), 71.2 (C-3'), 70.6

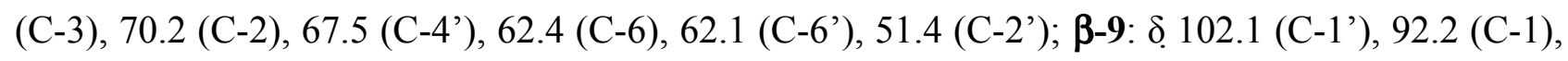
75.8, 74.3, 73.3 (C-3, C-4, C-5), 71.3 (C-5'), 71.2 (C-3'), 70.3 (C-2), 68.5 (C-4'), 62.8 (C-6), 62.1 (C-6'), 51.4 (C-2'); cluster of signals for both anomers: $\delta .171 .4-169.9(\mathrm{MeCO}), 23.2(\mathrm{MeCON})$, 21.2-20.8 (MeCOO). Anal Calcd for $\mathrm{C}_{28} \mathrm{H}_{39} \mathrm{NO}_{18}: \mathrm{C}, 49.63 ; \mathrm{H}, 5.80 ; \mathrm{N}, 2.07$. Found: C, 49.65; H, $5.83 ; \mathrm{N}, 2.08$.

\subsection{4-Methoxyphenyl}

3,4-O-isopropylidene-6-O-(1-methoxy-1-methylethyl)- $\beta$-Dtalopyranoside (11).

A mixture of $\mathbf{1 0}^{21}(320 \mathrm{mg}, 0.803 \mathrm{mmol})$, pre-dried 4-methylmorpholine $N$-oxide (NMO, 165 $\mathrm{mg}, 1.41 \mathrm{mmol})$ and $4 \AA$ powdered molecular sieves $(500 \mathrm{mg})$ in anhyd $\mathrm{CH}_{2} \mathrm{Cl}_{2}(15 \mathrm{~mL})$ was stirred for $30 \mathrm{~min}$ at room temp under argon atmosphere. Tetrapropylammonium perruthenate (TPAP, 14.1 $\mathrm{mg}, 0.04 \mathrm{mmol}$ ) was then added and the resulting mixture was stirred for $2 \mathrm{~h}$ at room temp until 
TLC (9:1 $\left.\mathrm{CH}_{2} \mathrm{Cl}_{2}-\mathrm{Me}_{2} \mathrm{CO}\right)$ revealed complete oxidation of $\mathbf{1 0}$. The mixture was filtered through a celite-silica gel-celite triple alternate pad, the filter was washed in the order with $\mathrm{CH}_{2} \mathrm{Cl}_{2}$ and EtOAc and the organic phase was concentrated under diminished pressure. The residue was dissolved in $\mathrm{MeOH}(15 \mathrm{~mL}), \mathrm{NaBH}_{4}(121.4 \mathrm{mg}, 3.21 \mathrm{mmol})$ was added and the mixture was stirred at $0{ }^{\circ} \mathrm{C}$. After $2 \mathrm{~h}$ TLC analysis (9:1 $\left.\mathrm{CH}_{2} \mathrm{Cl}_{2}-\mathrm{Me}_{2} \mathrm{CO}\right)$ showed the complete disappearance of the 2-uloside. Water $(8 \mathrm{~mL})$ was added and the resulting solution was stirred for additional $30 \mathrm{~min}$ and then extracted with $\mathrm{CH}_{2} \mathrm{Cl}_{2}(3 \times 30 \mathrm{~mL})$. The organic extracts were collected, dried $\left(\mathrm{MgSO}_{4}\right)$, filtered and concentrated under diminished pressure. Purification of the residue by flash chromatography over silica gel $\left(9: 1 \mathrm{CH}_{2} \mathrm{Cl}_{2}-\mathrm{Me}_{2} \mathrm{CO}+0.1 \% \mathrm{Et}_{3} \mathrm{~N}\right)$ afforded pure $11(259.2 \mathrm{mg}, 81 \%)$ as a clear syrup; $[\alpha]_{\mathrm{D}}-49.5$ (c 0.99, $\left.\mathrm{CHCl}_{3}\right) ; R_{\mathrm{f}} 0.43\left(9: 1 \mathrm{CH}_{2} \mathrm{Cl}_{2}-\mathrm{Me}_{2} \mathrm{CO}\right) ;{ }^{1} \mathrm{H} \mathrm{NMR}\left(\mathrm{CD}_{3} \mathrm{CN}\right): \delta 7.02,6.95$ (AA'XX' system, 4H, Ar-H), 5.08 (d, 1H, J1,2 $2.1 \mathrm{~Hz}, \mathrm{H}-1$ ), 4.37 (dd, 1H, $J_{3,4} 7.0 \mathrm{~Hz}, J_{2,3} 4.5 \mathrm{~Hz}$, H-3), 4.30 (dd, 1H, J4,5 $2.1 \mathrm{~Hz}, \mathrm{H}-4), 3.84$ (ddd, 1H, J,6a $6.9 \mathrm{~Hz}, J_{5,6 \mathrm{~b}} 5.2 \mathrm{~Hz}, \mathrm{H}-5$ ), 3.82 (dd, 1H, H-2), 3.73 (s, 3H, OMe), 3.59 (dd, 1H, J6a,6b 10.0 Hz, H-6a), 3.55 (dd, 1H, H-6b), 3.12 [s, 3H, $\left.\mathrm{C}(\mathrm{OMe}) \mathrm{Me}_{2}\right], 1.52,1.32\left(2 \mathrm{~s}\right.$, each $\left.3 \mathrm{H}, \mathrm{CMe} e_{2}\right), 1.28\left(\mathrm{~s}, 6 \mathrm{H}, \mathrm{C}(\mathrm{OMe}) M e_{2}\right) ;{ }^{13} \mathrm{C} \mathrm{NMR}\left(\mathrm{CD}_{3} \mathrm{CN}\right): \delta$ 155.9, 152.4 (Ar-C), 118.9, 115.3 (Ar-CH), $110.5\left(C \mathrm{Me}_{2}\right), 100.8$ [C(OMe) $\left.\mathrm{Me}_{2}\right], 99.5(\mathrm{C}-1), 73.9$ (C-3), 72.8 (C-4), 72.1 (C-5), 67.0 (C-2), 61.4 (C-6), 56.1 (OMe-1), 48.8 [C(OMe)Me $]$ ], 25.8, 25.4 $\left(\mathrm{CMe}_{2}\right), 24.7,24.6$ [C(OMe $\left.) \mathrm{Me}_{2}\right]$. Anal. Calcd for $\mathrm{C}_{20} \mathrm{H}_{30} \mathrm{O}_{8}: \mathrm{C}, 60.29 ; \mathrm{H}, 7.59$. Found: C, 60.47; H, 7.61 .

\subsection{4-Methoxyphenyl 2-O-(1-imidazolylsulfonyl)-3,4-O-isopropylidene-6-O-(1-methoxy-1- methylethyl)- $\beta$-D-talopyranoside (12).}

To a suspension of $60 \% \mathrm{NaH}$ in mineral oil (94 mg, $2.34 \mathrm{mmol})$ pre-washed with hexane under argon atmosphere and cooled to $0{ }^{\circ} \mathrm{C}$ a soln of $11(186.8 \mathrm{mg}, 0.469 \mathrm{mmol})$ in dry DMF (8 mL) was added. The mixture was stirred at $0{ }^{\circ} \mathrm{C}$ for $30 \mathrm{~min}$, cooled to $-30{ }^{\circ} \mathrm{C}$, treated with $\operatorname{Im}_{2} \mathrm{SO}_{2}(139 \mathrm{mg}$, $0.703 \mathrm{mmol}$ ) and further stirred at $-30{ }^{\circ} \mathrm{C}$. After $20 \mathrm{~min}$ TLC analysis (3:7 hexane-EtOAc) revealed the complete disappearance of the starting material. The reaction mixture was then cooled to -40 ${ }^{\circ} \mathrm{C}$, excess of $\mathrm{NaH}$ was destroyed by addition of $\mathrm{MeOH}(0.5 \mathrm{~mL})$ followed by $10 \mathrm{~min}$ stirring, then partitioned between $\mathrm{Et}_{2} \mathrm{O}(16 \mathrm{~mL})$ and crushed iced-water. The organic phase was separated, and the aq layer extracted with $\mathrm{Et}_{2} \mathrm{O}(2 \times 16 \mathrm{~mL})$. The collected organic phases were dried $\left(\mathrm{MgSO}_{4}\right)$, filtered and concentrated at diminished pressure. The flash chromatographic purification over silica gel of the reaction product $\left(2: 3\right.$ hexane-EtOAc $+0,1 \%$ of $\left.\mathrm{Et}_{3} \mathrm{~N}\right)$ gave pure $12(226.6 \mathrm{mg}, 92 \%)$ as a clear syrup; $[\alpha]_{\mathrm{D}}+4.2\left(\mathrm{c} 1.18, \mathrm{CHCl}_{3}\right) ; R_{\mathrm{f}} 0.22\left(2: 3\right.$ hexane-EtOAc); ${ }^{1} \mathrm{H} \mathrm{NMR}\left(\mathrm{CD}_{3} \mathrm{CN}\right): \delta 8.07(\mathrm{dd}$, 1H, $\left.J_{2,4} 1.3 \mathrm{~Hz}, J_{2,5} 0.8 \mathrm{~Hz}, \mathrm{Im}-\mathrm{H}-2\right), 7.50$ (dd, 1H, $\left.J_{4,5} 1.7 \mathrm{~Hz}, \mathrm{Im}-\mathrm{H}-4\right), 7.10$ (dd, 1H, Im-H-5), 6.83, 
6.72 (AA'XX' system, 4H, Ar-H), 5.03 (dd, 1H, $\left.J_{1,2} 1.0 \mathrm{~Hz}, J_{2,3} 5.5 \mathrm{~Hz}, \mathrm{H}-2\right), 4.93$ (d, 1H, H-1), $4.51\left(\mathrm{dd}, 1 \mathrm{H}, J_{3,4} 5.8 \mathrm{~Hz}, \mathrm{H}-3\right), 4.23$ (dd, $\left.1 \mathrm{H}, J_{4,5} 2.7 \mathrm{~Hz}, \mathrm{H}-4\right), 4.01$ (ddd, $1 \mathrm{H}, J_{5,6 \mathrm{a}} 7.4 \mathrm{~Hz}, J_{5,6 \mathrm{~b}} 4.7$ Hz, H-5), 3.71 (s, 3H, OMe), 3.65 (dd, 1H, J6a,6b 10.3 Hz, H-6a), 3.57 (dd, 1H, H-6b), 3.09 [s, 3H, $\mathrm{C}(\mathrm{OMe}) \mathrm{Me}_{2}$ ], 1.49, 1.33 (2s, each $\left.3 \mathrm{H}, \mathrm{CMe}\right), 1.29,1.28$ [2s, each $\left.3 \mathrm{H}, \mathrm{C}(\mathrm{OMe}) \mathrm{Me}_{2}\right]$; ${ }^{13} \mathrm{C} \mathrm{NMR}$ (CD ${ }_{3} \mathrm{CN}$ ): $\delta$ 156.3, 151.3 (Ar-C), 138.3 (Im-C-2), 131.1 (Im-C-5), 120.0 (Im-C-4), 118.6, 115.2 (Ar-CH), $111.4\left(\mathrm{CMe}_{2}\right), 100.8$ [C(OMe)Me 2$], 97.3$ (C-1), 79.3 (C-2), 73.8 (C-5), 71.9 (C-3), 71.4 (C-4), 60.7 (C-6), 56.1 (OMe-1), 48.8 [C(OMe) $\left.\mathrm{Me}_{2}\right], 25.9,25.4\left(\mathrm{CMe}_{2}\right)$, 24.7, 24.6 [C(OMe)Me $\mathrm{Ce}_{2}$. Anal. Calcd for $\mathrm{C}_{23} \mathrm{H}_{32} \mathrm{~N}_{2} \mathrm{O}_{10} \mathrm{~S}: \mathrm{C}, 52.26 ; \mathrm{H}, 6.10 ; \mathrm{N}, 5.30 ; \mathrm{S}, 6.07$. Found: $\mathrm{C}, 52.46 ; \mathrm{H}, 6.18 ; \mathrm{N}$, $5.33 ; \mathrm{S}, 6.11$.

\subsection{4-Methoxyphenyl 2-azido-2-deoxy-3,4-O-isopropylidene-6-O-(1-methoxy-1-methylethyl)- $\beta$-D-galactopyranoside (13).}

A soln of $12(91.2 \mathrm{mg}, 0.173 \mathrm{mmol})$ and $\mathrm{NaN}_{3}(22.5 \mathrm{mg}, 0.345 \mathrm{mmol})$ in dry DMF (4 mL) was stirred at $100{ }^{\circ} \mathrm{C}$ under argon atmosphere. After $2 \mathrm{~h}$ the mixture was cooled to room temp and partitioned between satd aq $\mathrm{NaHCO}_{3}(8 \mathrm{~mL})$ and $\mathrm{Et}_{2} \mathrm{O}(15 \mathrm{~mL})$. The organic phase was separated and the aq layer extracted with $\mathrm{Et}_{2} \mathrm{O}(3 \times 15 \mathrm{~mL})$. The organic extracts were collected, dried $\left(\mathrm{MgSO}_{4}\right)$, filtered and concentrated at diminished pressure. Purification of the residue by flash chromatography over silica gel, eluting with 7:3 hexane-EtOAc $+0.1 \%$ of $\mathrm{Et}_{3} \mathrm{~N}$, gave pure 13 (71 $\mathrm{mg}, 96 \%)$ as a clear syrup; $[\alpha]_{\mathrm{D}}+35.5\left(\mathrm{c} 0.67, \mathrm{CHCl}_{3}\right) ; R_{\mathrm{f}} 0.32$ (7:3 hexane-EtOAc); ${ }^{1} \mathrm{H}$ NMR $\left(\mathrm{CD}_{3} \mathrm{CN}\right.$ ): $\delta$ 7.03, 6.92 (AA'XX' system, 4H, Ar-H), 4.80 (d, 1H, $J_{1,2} 8.6 \mathrm{~Hz}, \mathrm{H}-1$ ), 4.19 (dd, 1H, $J_{3,4}$ $\left.5.3 \mathrm{~Hz}, J_{4,5} 2.0 \mathrm{~Hz}, \mathrm{H}-4\right), 4.02\left(\mathrm{ddd}, 1 \mathrm{H}, J_{5,6 \mathrm{a}} 7.0 \mathrm{~Hz}, J_{5,6 \mathrm{~b}} 5.2 \mathrm{~Hz}, \mathrm{H}-5\right), 3.98$ (dd, 1H, $J_{2,3} 7.5 \mathrm{~Hz}$, H-3), 3.75 (s, 3H, OMe), 3.64 (dd, 1H, Ja, 10.0 Hz, H-6a), 3.63 (dd, 1H, H-2), 3.59 (dd, 1H, $\mathrm{H}-6 \mathrm{~b}), 3.12$ [s, 3H, C(OMe) $\left.\mathrm{Me}_{2}\right], 1.52,1.32$ (2s, each 3H, $\mathrm{CMe}$ ), 1.31, 1.30 [2s, each 3H, $\left.\mathrm{C}(\mathrm{OMe}) \mathrm{Me}_{2}\right] ;{ }^{13} \mathrm{C} \mathrm{NMR}\left(\mathrm{CD}_{3} \mathrm{CN}\right): \delta$ 156.5, 151.8 (Ar-C), 119.1, 115.5 (Ar-CH), $111.1\left(\mathrm{CMe}_{2}\right)$, 101.4 (C-1), 100.8 [C(OMe)Me $]$ ], 77.9 (C-3), 73.9 (C-4), 73.3 (C-5), 66.0 (C-2), 60.9 (C-6), 56.1 (OMe-1), $48.8\left[\mathrm{C}(\mathrm{OMe}) \mathrm{Me}_{2}\right], 28.4,26.4\left(\mathrm{CMe}_{2}\right), 24.7,24.6$ [C(OMe) $\left.\mathrm{Me}_{2}\right]$. Anal. Calcd for $\mathrm{C}_{20} \mathrm{H}_{31} \mathrm{~N}_{3} \mathrm{O}_{7}$ : C, 56.46; H, 7.34; N, 9.88. Found: C, 56.48; H, 7.38; N, 9.91.

\subsection{4-Methoxyphenyl 2-azido-2-deoxy-3,4,6-tri- $O$-acetyl- $\beta$-D-galactopyranoside (14).}

A soln of $13(68 \mathrm{mg}, 0.16 \mathrm{mmol})$ in $80 \%$ aq $\mathrm{AcOH}(3 \mathrm{~mL})$ was stirred at $80{ }^{\circ} \mathrm{C}$ for $2 \mathrm{~h}$, then concentrated at reduced pressure and co-evaporated with toluene $(3 \times 8 \mathrm{~mL})$. The residue was dissolved in a 2:1 pyridine- $\mathrm{Ac}_{2} \mathrm{O}$ soln $(3 \mathrm{~mL})$ and the resulting soln was stirred at room temp for $2 \mathrm{~h}$ 
and then co-evaporated with toluene $(3 \times 8 \mathrm{~mL})$. The flash cromatographic purification over silica gel (7:3 hexane-EtOAc) afforded pure $14(67.8 \mathrm{mg}, 97 \%)$ as a clear syrup; $[\alpha]_{\mathrm{D}}+6.02$ (c 0.83, $\left.\mathrm{CHCl}_{3}\right) ; R_{\mathrm{f}} 0.56$ (2:3 hexane-EtOAc); ${ }^{1} \mathrm{H}$ NMR $\left(\mathrm{CD}_{3} \mathrm{CN}\right): \delta 7.03,6.90$ (AA'XX' system, 4H, Ar-H), 5.33 (dd, 1H, J3,4 $\left.3.4 \mathrm{~Hz}, J_{4,5} 1.0 \mathrm{~Hz}, \mathrm{H}-4\right), 5.00$ (d, 1H, $J_{1,2} 8.1 \mathrm{~Hz}, \mathrm{H}-1$ ), 4.92 (dd, 1H, $J_{2,3} 10.8 \mathrm{~Hz}$, H-3), 4.24-4.04 (m, 3H, H-6a, H-6b, H-5), 3.98 (dd, 1H, H-2), 3.75 (s, 3H, OMe), 2.21, 2.00, 1.99 $(3 \mathrm{~s}$, each $3 \mathrm{H}, 3 \times \mathrm{MeCO}) ;{ }^{13} \mathrm{C} \mathrm{NMR}\left(\mathrm{CD}_{3} \mathrm{CN}\right): \delta 171.2,171.1,170.7(3 \times \mathrm{MeCO}), 156.6,151.6(\mathrm{Ar}-$ C), 119.0, 115.6 (Ar-CH), 101.6 (C-1), 71.9 (C-5), 71.8 (C-3), 67.5 (C-4), 62.4 (C-6), 61.6 (C-2), 56.1 (OMe-1), 20.8 (MeCO). Anal. Calcd for $\mathrm{C}_{19} \mathrm{H}_{23} \mathrm{~N}_{3} \mathrm{O}_{9}$ : C, 52.17; H, 5.30; N, 9.61. Found: C, $52.20 ; \mathrm{H}, 5.33 ; \mathrm{N}, 9.63$.

\subsection{2-Azido-2-deoxy-3,4,6-tri- $O$-acetyl- $\alpha, \beta$-D-galactopyranose (15).}

To a soln of 14 (67 mg, $0.153 \mathrm{mmol})$ in 3:1 $\mathrm{Me}_{2} \mathrm{CO}$-water (6 mL), CAN (587 mg, $\left.1.07 \mathrm{mmol}\right)$ was added at $0{ }^{\circ} \mathrm{C}$, the soln was warmed to room temp and stirred for $30 \mathrm{~min}$. The soln was concentrated to $3 \mathrm{~mL}$, diluted with $\mathrm{CH}_{2} \mathrm{Cl}_{2}(25 \mathrm{~mL})$, washed with sat aq $\mathrm{NaHCO}_{3}(2 \times 15 \mathrm{~mL})$, dried $\left(\mathrm{MgSO}_{4}\right)$, filtered and concentrated under diminished pressure. Purification of the residue by flash chromatography over silica gel (7:3 hexane-EtOAc) afforded known $\mathbf{1 5}^{23}(41 \mathrm{mg}, 81 \%)$ as a clear syrup constituted $\left(\mathrm{NMR}, \mathrm{CDCl}_{3}\right)$ by a mixture of $\alpha$ - and $\beta$-anomers in $3: 2$ ratio calculated on the basis of the relative intensities of $\mathrm{C}-1$ signals ( $\delta 92.3$ and 96.3 , respectively); $[\alpha]_{\text {Dinizial }}+61.7,[\alpha]_{\text {Do }}$ +55.5 (c 0.91, MeOH); $R_{\mathrm{f}} 0.20$ (7:3 hexane-EtOAc); ${ }^{1} \mathrm{H}$ NMR $\left(\mathrm{CDCl}_{3}\right)$ of $\boldsymbol{\alpha}-15: \delta 5.43\left(\mathrm{~d}, 1 \mathrm{H}, J_{1,2}\right.$ $3.3 \mathrm{~Hz}, \mathrm{H}-1), 5.37$ (dd, 1H, $\left.J_{2,3} 10.0 \mathrm{~Hz}, J_{3,4} 3.2 \mathrm{~Hz}, \mathrm{H}-3\right), 4.47$ (m, 1H, H-5), 3.74 (dd, 1H, H-2), 3.91 (bs, 1H, OH-1); $\beta$-15: $\delta 4.83$ (dd, 1H, J,3 10.9 Hz, J3,4 $3.3 \mathrm{~Hz}, \mathrm{H}-3$ ), 4.72 (dd, 1H, $J_{1,2} 7.8 \mathrm{~Hz}$, $\left.J_{1, \text { OH }} 5.3 \mathrm{~Hz}, \mathrm{H}-1\right), 3.91$ (m, 1H, H-5), 3.67 (dd, 1H, H-2), 4.58 (d, 1H, OH-1); cluster of signals for both anomers: $\delta 5.45$ (m, 1H, H-4), 4.08-4.22 (m, 2H, H-6a, H-6b); 2.20-1.97 (m, 9H, 3 x MeCO);

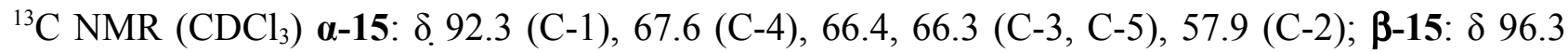
(C-1), 71.1 (C-3), 70.8 (C-5), 68.3 (C-4), 61.9 (C-2); cluster of signals for both anomers: $\delta$ 170-7-170.0 (MeCO), 61.7, 61.3 (C-6), 20.7-20.5 (MeCO). Anal. Calcd for $\mathrm{C}_{12} \mathrm{H}_{19} \mathrm{~N}_{3} \mathrm{O}_{8}$ : C, 43.24; H, 5.75; N, 12.61. Found: C, 43.26; H, 5.76; N, 12.62 .

\subsection{2-Azido-2-deoxy-1,3,4,6-tetra- $O$-acetyl- $\alpha, \beta$-D-galactopyranose (16).}

A soln of $15(32 \mathrm{mg}, 0.10 \mathrm{mmol})$ in a $2: 1$ pyridine- $\mathrm{Ac}_{2} \mathrm{O}$ soln $(2 \mathrm{~mL})$ was stirred at room temp for $15 \mathrm{~h}$ and then co-evaporated with toluene $(3 \times 8 \mathrm{~mL})$. The flash cromatographic purification over silica gel (1:1 hexane-EtOAc) afforded pure 16 (34 mg, 95\%) as a clear syrup, constituted 
$\left(\mathrm{NMR}, \mathrm{CDCl}_{3}\right)$ by a mixture of $\alpha$ - and $\beta$-anomers in 1:1 ratio calculated on the basis of the relative integral of H-1 signals ( $\delta 6.28$ and 5.50, respectively); $R_{\mathrm{f}} 0.65$ (1:1 hexane-EtOAc); $[\alpha]_{\mathrm{D}}+49.7$ (c $\left.0.92, \mathrm{CHCl}_{3}\right) ; \mathrm{Lit}^{24}[\alpha]_{\mathrm{D}}+36.7\left(\mathrm{c} 1.6, \mathrm{CHCl}_{3}\right)$ for a $2: 3$ mixture of $\alpha$ - and $\beta$-anomers; NMR data were in full accordance with those reported. ${ }^{24}$

\section{Acknowledgement}

This research was supported by Ministero dell'Università e della Ricerca (MIUR, Rome, Italy) in the frame of the national project COFIN 2006.

\section{References}

1. Attolino, E.; Bonaccorsi, F.; Catelani, G.; D'Andrea, F. Carbohydr. Res., 2008, 343, 2545-2556.

2. Levene, P.A.; La Forge, F.B. J. Biol. Chem. 1914, 18, 123-130.

3. Jeanloz, R.W. In The Carbohydrates. Chemistry and Biochemistry, Pigman, W.; Horton. D. Eds. Academic Press, New York, 1970, Vol IIB, pp 590-625.

4. Hanisch, F.-G.; Müller, S. Glycobiology, 2000, 10, 439-449.

5. Sharon, N. In Complex Carbohydrates. Their Chemistry, Biosynthesis, and Functions, AddisonWesley Publ. 1975.

6. Krist, P.; Herkommerová-Rajnochová, E.; Rauvolfová, J.; Semeňuk, T.; Vavrušková, P.; Pavlíček J.; Bezouška, K., Petruš, L.; Křen. V. Biochem. Biophys. Res. Commun. 2001, 287, 11-20.

7. Selected representative reports: (a) Gross, P.H.; Du Bois, F.; Jeanloz, R.W. Carbohydr. Res. 1967, 4, 244-248; (b) Hill, J.; Hough, L. Carbohydr. Res. 1968, 8, 398; (c) Fukuda, Y.; Sasai, H.; Suami, T. Bull. Soc. Chim. Jpn. 1982, 55, 1574; (d) McGeary, R.P.; Wright, K.; Toth, I. J. Org. Chem. 2001, 66, 5102; (e) Misra, A.K.; Fukuda, M.; Hindsgault, O. Bioorg. Med. Chem. Lett. 2001, 11, 2667-2669; (f) Ito, N.; Tokuda, Y.; Ohba, S.; Sugai, T. Bull. Soc. Chim. Jpn. 2004, 77, 1181-1186; (g) Hederos, M.; Konradsson, P.J. J. Carbohydr. Chem. 2005, 24, 297-320.

8. Paulsen, H.; Koláŕ, Č.; Stenzel, W. Angew. Chem. 1976, 88, 1532-1568.

9. Lemieux, R.U.; Ratcliffe, R.M. Canad. J. Chem. 1979, 57, 1244-1251.

10. Griffith, D.A.; Danishefsky, S.J. J. Am. Chem. Soc. 1990, 112, 5811-5819.

11. Liu, J.; Gin, D.Y. J. Am. Chem. Soc. 2002, 124, 9789-9797.

12. (a) Kuhn, R.; Kirschenlohr, W. Angew. Chem. 1955, 67, 786; (b) Brossmer, R. Methods Carbohydr. Chem. 1962, 1, 216-221.

13. Wrodnigg, T.M.; Lundt, I.; Stütz, A. E. J. Carbohydr. Chem. 2006, 25, 33-41. 
14. Attolino, E.; Bonaccorsi, F.; Catelani, G.; D’Andrea, F.; Křenel, K.; Bešouska, K.; Křen, V. J. Carbohydr. Chem. 2008, 27, 156-171.

15. Van den Nieuwenhof, I.M.; Schiphorst, W.E.; Van den Eijnden, D.H. FEBS letters, 459, 1999, 377-80.

16. Neeleman, A.P.; Van den Eijnden, D.H. Proc. Natl. Acad. Sci. USA 1996, 93, 10111-10116.

17. Attolino, E.; Catelani, G.; D'Andrea, F. Eur. J. Org. Chem. 2006, 23, 5279-5292.

18. (a) David, S.; Malleron, A.; Dini, C. Carbohydr. Res. 1989, 188, 193-200; (b) Vatèle, J.-M.; Hanessian, S. In Preparative Carbohydrate Chemistry, Hanessian, S. Editor, M. Dekker (New York) 1997, 127-149.

19. Bock, K.; Thøgersen, H. Ann. Rep. NMR Spectrosc. 1982, 13, 1-57.

20. Izumi, K. Carbohyd. Res. 1987, 170, 19-25.

21. (a) Thijssen, M.J.L.; van Rijswijk, M.N.; Kamerling, J.P.; Vliegenthart, J.F. G. Carbohyd. Res. 306, 1998, 93-109; (b) Jacquinet, J-C. Carbohyd. Res. 2006, 341, 1630-1644.

22. Zhang, Z.; Magnusson, G. J. Org. Chem. 1996, 61, 2383-2393.

23. Grundler, G.; Schmidt, R. Liebigs Ann. Chem. 1984, 1826-1847.

24. Vasella, A.; Witzig, C.; Chiara, J.-L.; Martin-Lomas, M. Helv. Chim. Acta 1991, 74, 2073-2077.

25. For glycosyl trichloroacetimidates: Payne, R.J.; Ficht, S.; Tang, S.; Brik, A.; Yang, Y.-Y.; Case, D.A.; Wong, C.-H. J. Am. Chem. Soc, 2007, 129, 13527-13536; for glycosyl N-phenyltrifluoroacetimidates: Adinolfi, M.; Iadonisi, A.; Ravidà, A.; Schiattarella, M. J. Org. Chem. 70, 2005, 5316-5319; for a glycosyl fluoride: Grigalevicius, S.; Chierici, S.; Renaudet, O.; Lo-Man, R.; Dériaud, E.; Leclerc, C.; Dumy, P. Bioconjugate Chem. 2005, 16, 1149-1159; for a glycosyl diphenylphosphate: Tsuda, T.; Nakamura, S.; Hashimoto, S. Tetrahedron 60, 2004, 10711-10737. 
Table 1. ${ }^{13} \mathrm{C}$ NMR chemical shifts of $\alpha$ - and $\beta$-1a and related compounds ${ }^{\mathrm{a}}$

\begin{tabular}{ccccccccccccc}
\hline Compound & $\mathbf{C - 1}$, & $\mathbf{C - 2}$ & $\mathbf{C - 3}$ & $\mathbf{C - 4}$ & $\mathbf{C - 5}$ & $\mathbf{C - 6}$ & $\mathbf{C - 1}$ & $\mathbf{C - 2}$ & $\mathbf{C - 3}$ & $\mathbf{C - 4}$ & $\mathbf{C - 5}$ & $\mathbf{C - 6}$ \\
\hline $\boldsymbol{\alpha}$-lactose & 103.6 & 72.0 & 73.5 & 69.5 & 76.2 & 62.0 & 92.7 & 72.2 & 72.4 & 79.3 & 71.0 & 61.0 \\
$\boldsymbol{\beta}$-lactose & 103.6 & 72.0 & 73.5 & 69.5 & 76.2 & 62.0 & 96.6 & 74.8 & 75.3 & 79.2 & 75.6 & 61.1 \\
$\mathbf{M e} \boldsymbol{\beta}$-D-GalNAcp & 103.1 & 53.0 & 71.9 & 68.6 & 75.8 & 61.7 & & & & & & \\
$\boldsymbol{\alpha - 1 a}$ & 104.5 & 55.4 & 73.5 & 70.5 & 78.2 & 63.8 & 94.6 & 74.3 & 73.8 & 82.1 & 72.6 & $62.9^{\mathrm{b}}$ \\
$\boldsymbol{\beta}-1 \mathbf{a}$ & 104.5 & 55.4 & 73.5 & 70.5 & 78.2 & 63.8 & 98.5 & 76.5 & $77.4^{\mathrm{b}}$ & 81.9 & $77.3^{\mathrm{b}}$ & $63.0^{\mathrm{b}}$ \\
\hline
\end{tabular}

${ }^{\mathrm{a}}$ Spectra taken in $\mathrm{D}_{2} \mathrm{O}$. Internal reference: 1,4-dioxane for lactose (Ref. 19) and Me- $\beta$-D-GalNAc (Ref. 20), TMSP for 1a.

${ }^{\mathrm{b}}$ Assigments may be reversed. 